\title{
A Case of Takayasu's Arteritis Associated with Human Leukocyte Antigen A24 and B52 Following Resolution of Ulcerative Colitis and Subacute Thyroiditis
}

\author{
Yoshiro Horai, Tomoya Miyamura, Karin Shimada, Soichiro Takahama, \\ Rumi Minami, Masahiro Yamamoto and Eiichi Suematsu
}

\begin{abstract}
A 46-year-old female with a past history of ulcerative colitis (UC) was diagnosed with subacute thyroiditis (SAT), which improved with prednisolone (PSL) treatment $(60 \mathrm{mg} / \mathrm{day})$. The dose of PSL was gradually decreased, however upper back and neck pain and chest discomfort developed. The patient was diagnosed with Takayasu's arteritis (TA) based on wall thickening and luminal narrowing of the left common carotid artery and the left subclavian artery. The result of human leukocyte antigen typing analysis was A24 and B52 positive. These findings suggested that common genetic factors may be important for the etiology of TA, UC and SAT. This is the first report of TA that developed following UC and SAT.
\end{abstract}

Key words: Takayasu's arteritis, ulcerative colitis, subacute thyroiditis, human leukocyte antigen

(Intern Med 50: 151-154, 2011)

(DOI: 10.2169/internalmedicine.50.4385)

\section{Introduction}

Takayasu's arteritis (TA) is a large vessel vasculitis named after a Mikito Takayasu, a Japanese ophthalmologist who reported the first case of TA in 1908 (1). Although the cause of TA is still unclear, genetic factors are considered to play important roles in the pathogenesis of this disease, as specific types of human leukocyte antigen (HLA) are common among patients with TA (2). Ulcerative colitis (UC), an inflammatory bowel disease, as well as TA, are considered autoimmune diseases. There have been quite a few reports describing cases of TA complicated with UC, which suggests that the two diseases might have common genetic factors. TA associated with subacute thyroiditis (SAT) is rare, with only two cases being reported (3). SAT has been suggested to potentially associate with certain HLA haplotypes like TA and UC $(4,5)$. There are currently no reports of UC, SAT and TA developing in the same patient. In this study, we describe a 46-year-old female with TA following resolution of SAT in a patient with past history of UC.

\section{Case Report}

A 46-year-old female developed UC when she was 22 years old that was treated with oral immunosuppressants and enemas for approximately 10 years. She was diagnosed in June 2009 with SAT following pyrexia, neck pain and high serum thyroid hormone levels. Administration of $60 \mathrm{mg} /$ day of prednisolone (PSL) was started and symptoms improved. The dose of PSL was decreased slowly without relapse. Administration of PSL was completely stopped in November 2009. However, neck and back pain developed in January 2010, which was complicated by chest discomfort. The patient then experienced nocturnal dyspnea and visited a local clinic. TA was suspected based on the presence of decreased pulsation in the left radial artery, laterality of upper arm blood pressure and heart murmur. She was referred to our department in April 2010.

When examined at our department following referral, her pulse rate was regular (73 beats per minute). Her height was $165.6 \mathrm{~cm}$ and body weight was $72.4 \mathrm{~kg}$. Blood pressure was $123 / 56 \mathrm{mmHg}$ in the right arm and $106 / 50 \mathrm{mmHg}$ in the

Department of Internal Medicine and Rheumatology, Clinical Research Center, National Hospital Organization Kyushu Medical Center, Japan Received for publication August 18, 2010; Accepted for publication October 6, 2010 Correspondence to Dr. Yoshiro Horai, yoshirohorai0518@qmed.hosp.go.jp 


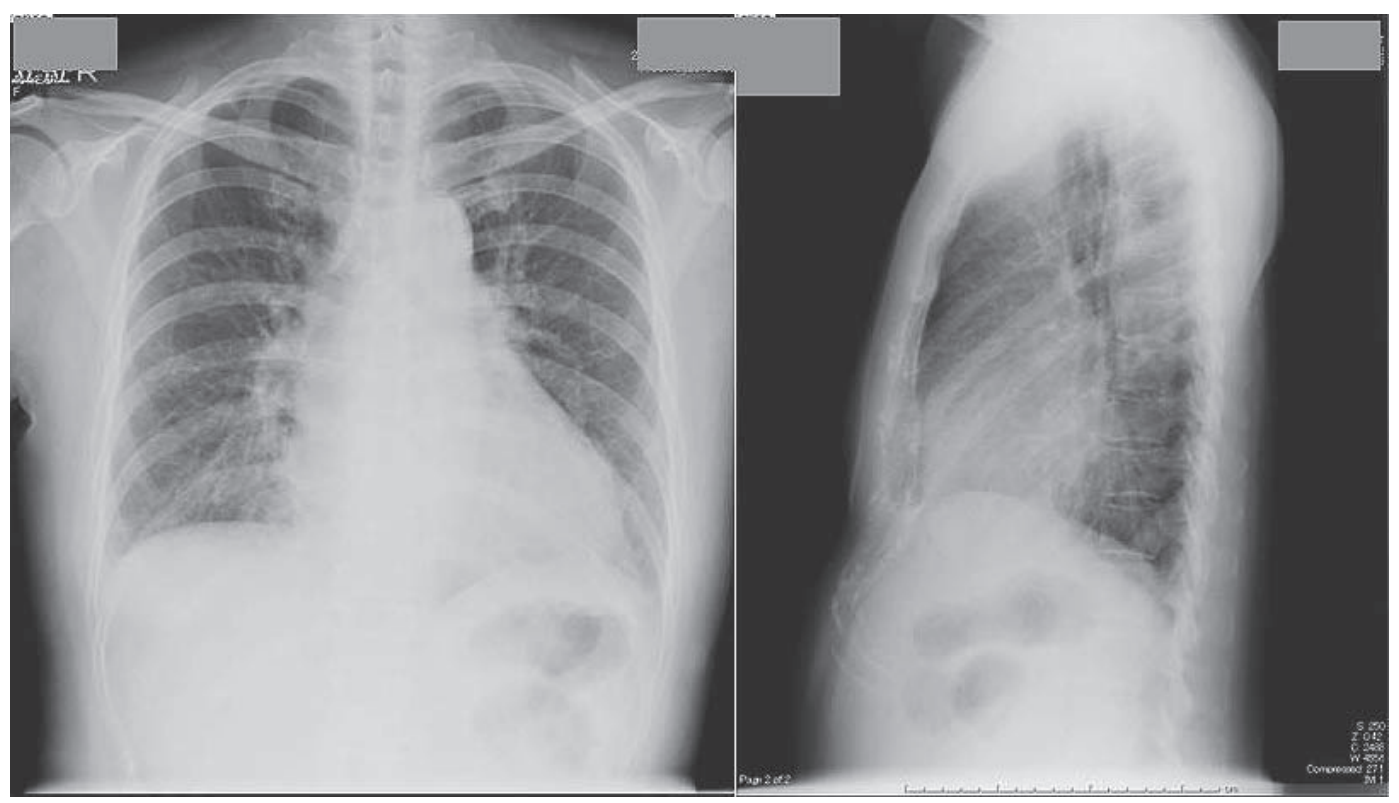

Figure 1. Chest X-ray obtained at our department showing cardiomegaly (cardiothoracic ratio $56 \%$ ) and lung congestion.

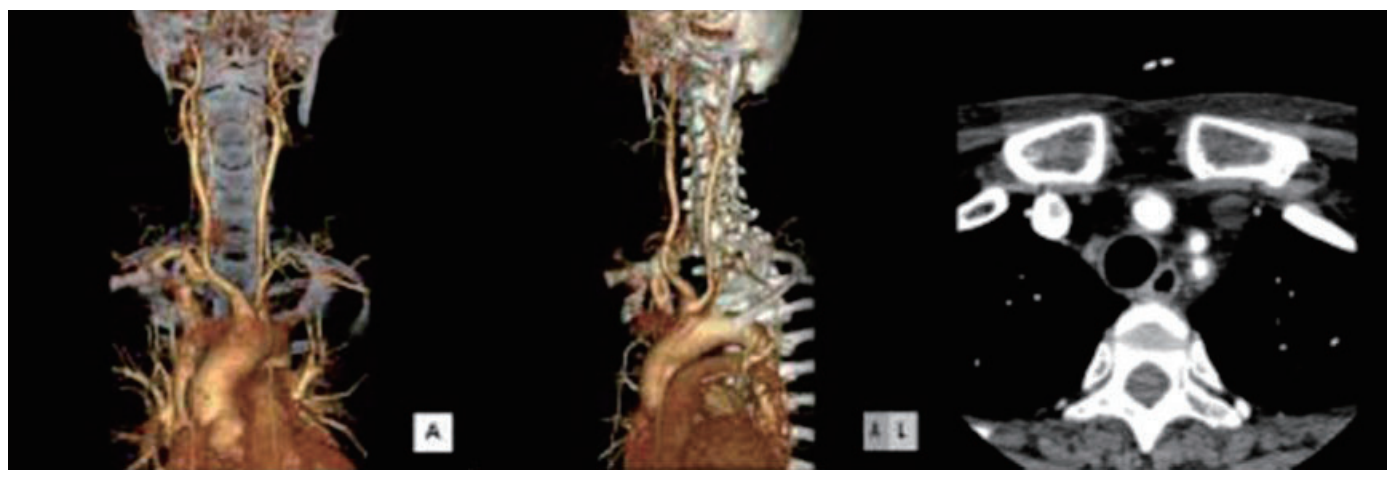

Figure 2. Three-dimensional computed tomography showing wall thickening and luminal narrowing of the left common carotid artery and the left subclavian artery.

left arm. Bruits were audible in the left neck and a grade 3 aortic diastolic murmur was present at the third left sternal border. No abdominal bruits were heard and no neurological abnormalities were noted. Results of laboratory tests were: white blood cell count, 9,000/ $\mu \mathrm{L}$ (neutrophils 60.6\%, lymphocytes $30.0 \%$, monocytes $4.7 \%$, eosinophils $2.3 \%$, basophils $0.1 \%$ ); hemoglobin, $12.4 \mathrm{~g} / \mathrm{dL}$; platelet count, 372,000/ $\mu \mathrm{L}$; C-reactive protein, $2.60 \mathrm{mg} / \mathrm{dL}$; erythrocyte sedimentation rate, $31 \mathrm{~mm} /$ hour; blood urea nitrogen, $4 \mathrm{mg} / \mathrm{dL}$; serum creatinine, $0.5 \mathrm{mg} / \mathrm{dL}$; D-dimer, $1.7 \mu \mathrm{g} / \mathrm{dL}$. Brain natriuretic peptide level was extremely high $(748.4 \mathrm{pg} / \mathrm{mL})$, but thyroid stimulating hormone (TSH) and thyroid hormone levels (fT4) were normal (TSH, $4.610 \mu \mathrm{IU} / \mathrm{mL}$; fT4, $1.54 \mathrm{ng} / \mathrm{dL}$ ). HLA typing analysis revealed positivity for A24 and B52. A chest X-ray revealed cardiomegaly (cardiothoracic ratio $56 \%$ ), bilateral pleural effusion and lung congestion, compatible with congestive heart failure (Fig. 1). A 12-lead electrocardiogram revealed no abnormal findings. An echocardiogram revealed dilatation of the left ventricular cavity
(LVDd, $63 \mathrm{~mm}$; LVDd $51 \mathrm{~mm}$ ) and left atrial cavity (43 $\mathrm{mm}$ ), dilatation of the aorta, low ejection fraction (39\%), mild mitral regurgitation and moderate to severe aortic regurgitation. Three-dimensional computed tomography (CT) revealed wall thickening and luminal narrowing of the left common carotid artery and the left subclavian artery, which is compatible with TA (Fig. 2). An aortography revealed dilatation of the aortic root, aortic regurgitation and decreased ventricular contraction (ejection fraction $45.7 \%$, cardiac output 7.7 L/min, cardiac index $4.3 \mathrm{~L} / \mathrm{min} / \mathrm{m}^{2}$; Fig. 3). A coronary angiography showed no significant coronary artery stenosis.

A diagnosis of TA was made based on CT findings and elevated CRP and ESR levels, which meet the criteria proposed by the Japanese Ministry of Health and Welfare in 1992. Patient presentation also satisfied four of the six criteria for 1990 classification of TA by the American College of Rheumatology. These include the presence of decreased branchial artery pulse, greater than $10 \mathrm{mmHg}$ difference in 
A.

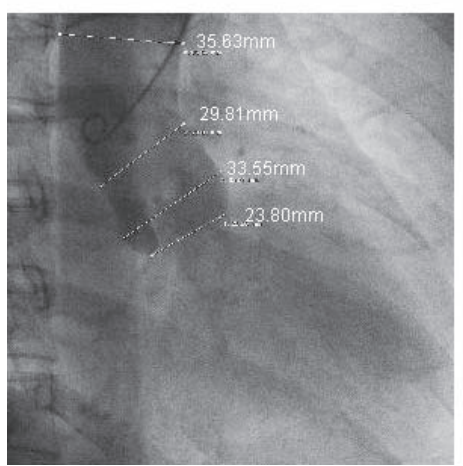

B.

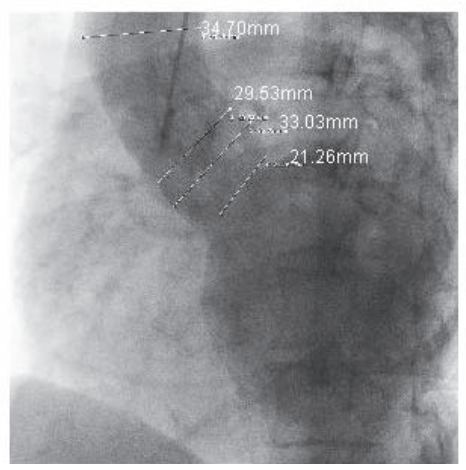

C.

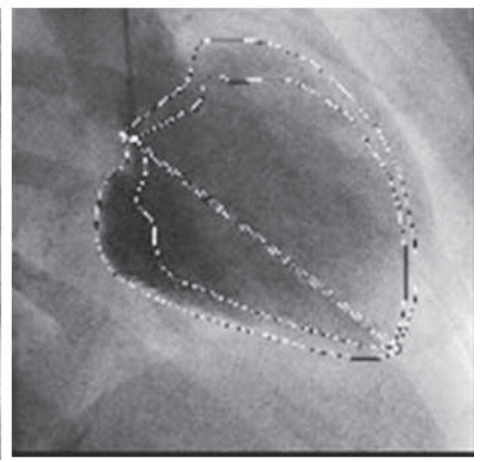

Figure 3. Aortography (A) showing dilatation of the aortic root, aortic regurgitation, decreased ventricular contraction, (B) severe aortic regurgitation, and (C) left ventriculography (ejection fraction $45.7 \%$, cardiac output $7.7 \mathrm{~L} / \mathrm{min}$, cardiac index $4.3 \mathrm{~L} / \mathrm{min} / \mathrm{m}^{2}$ ).

systolic blood pressure between arms, a bruit over the subclavian arteries or the aorta and arteriographic evidence of narrowing or occlusion of the entire aorta, its primary branches, or large arteries in the proximal upper or lower extremities (6). Treatment with furosemide, enalapril and a $\beta$-adrenergic antagonist was initiated for the management of congestive heart failure. In addition, digoxin and warfarin were also administered due to the development of paroxysmal atrial fibrillation. Although her symptoms of heart failure improved following treatment, high CRP levels persisted. Administration of PSL (30 mg/day) was begun and CRP levels immediately became normalized. PSL dose was decreased to $25 \mathrm{mg} /$ day around two weeks after the initial treatment. Three months after referral to our department, aortic valve replacement was successfully performed. The dose of PSL has been gradually decreased without relapse.

\section{Discussion}

TA is a large vessel vasculitis affecting the aorta and its first branches, and is pathologically characterized by granulomatous necrotizing vasculitis with giant cells. Because certain HLA types, e.g. A24, B52, and DR2, are common among patients with TA in Japanese (2), TA is considered an autoimmune vasculitis. Patients with TA positive for these typical haplotypes are known to have a tendency to be rapidly progressive and refractory to steroid therapy (7).

$\mathrm{UC}$ is an inflammatory bowel disease mainly affecting the colonic mucosa. Iizuka et al estimated that around $0.065 \%$ of UC patients were complicated with TA given that 39 cases of TA complicated with UC were reported, and about 60,000 patients with UC were registered in Japan as of 1995 (8). Based on the same analysis method, it is estimated that $0.783 \%$ of TA patients were complicated with UC, with 4,980 UC patients being registered in Japan since 1996. Analysis of patients with TA complicated with UC revealed a tendency to be positive for specific types of HLA. Numano et al analyzed reports of 12 patients with TA complicated with UC. Ten of the 12 patients were positive for
HLA-B52 (2). Morita et al analyzed nine patients with TA complicated with UC. Seven patients were positive for HLA-B52 and five were positive for A24. In addition, there were five HLA-A24 and B52 double-positive patients, as seen here with the present case (9). Although the patient number is small, this report suggests that patients who are positive for HLA-A24 and B52 may have a higher risk of concomitant development of TA and UC.

An interesting feature of this TA case is that the patient's clinical course was associated with SAT, in addition to UC. One other case with the combination of TA, UC and another autoimmune disease (ankylosing spondylitis) has been reported (10). Like TA and UC, SAT has been thought to be associated with certain types of HLA, especially B35 (4). Hokama et al reported a case of TA associated with UC, which was positive for HLA-B35, not B52 (11). In addition, Ohsako et al reported, following the analysis of 56 six Japanese patients with SAT, that SAT is associated with HLAB35 and B67 (5). These reports suggest that genetic factors may be involved in the development of SAT, as well as TA. In this case, the fact that TA developed less than one year after SAT improvement suggests that TA was triggered by SAT. However, the possibility that SAT and TA developed concurrently is not improbable as neck pain and pyrexia are early signs of TA. Early investigation with imaging methods, such as three-dimensional CT and aortography, seems to be important for diagnosis, especially for young female patients with fever of unknown origin and symptoms suggesting TA. Reported cases of TA associated with SAT are extremely rare. To the best of our knowledge, only two cases of this combination have been described, both by Ohta et al (3). The lack of TA case reports associated with SAT means that the interaction between these conditions remains unclear. Further studies are necessary to clarify the association between TA and SAT.

This is the first report describing the ordered development of UC, SAT and then TA. As the three diseases have autoimmune factors in common, it is therefore necessary to recognize that the development of one of the three diseases 
may trigger the other two disorders. This case opens for consideration the role of genetic factors in the pathogenesis of UC, SAT and TA.

The authors state that they have no Conflict of Interest (COI).

\section{References}

1. Numano F. The story of Takayasu arteritis. Rheumatology 41: 103-106, 2002.

2. Numano F, Miyata T, Nakajima T. Ulcerative colitis, Takayasu arteritis and HLA. Intern Med 35: 521-522, 1996.

3. Ohta Y, Ohya Y, Fujii K, et al. Inflammatory diseases associated with Takayasu's arteritis. Angiology 54: 339-344, 2003.

4. Rubin RA, Guay AT. Susceptibility to subacute thyroiditis is genetically influenced: familial occurrence in identical twins. Thyroid 1: 157-161, 1991.

5. Ohsako N, Tamai H, Sudo T, et al. Clinical characteristics of sub- acute thyroiditis classified according to human leukocyte antigen typing. J Clin Endocrinol Metab 80: 3653-3656, 1995.

6. Arend WP, Michel BA, Bloch DA, et al. The American College of Rheumatology 1990 criteria for the classification of Takayasu arteritis. Arthritis Rheum 33: 1129-1134, 1990.

7. Numano F, Okawara M, Inomata H, Kobayashi Y. Takayasu's arteritis. Lancet 356: 1023-1025, 2000.

8. Iizuka B, Yamagishi N, Honma N, Hayashi N. Cardio vascular disease associated with ulcerative colitis. Nippon Rinsho 57: 134139, 1999 (in Japanese).

9. Morita Y, Yamamura M, Suwaki K, et al. Takayasu's arteritis associated with ulcerative colitis; Genetic factors in this association. Intern Med 35: 574-578, 1996.

10. Aoyagi S, Akashi H, Kawara T, et al. Aortic root replacement for Takayasu arteritis associated with ulcerative colitis and ankylosing spondylitis. Jpn Circ J 62: 64-68, 1998.

11. Hokama A, Kinjo F, Arakaki T, et al. Pulseless hematochezia: Takayasu's arteritis associated with ulcerative colitis. Intern Med 42: 897-898, 2003.

(C) 2011 The Japanese Society of Internal Medicine http://www.naika.or.jp/imindex.html 